\title{
Effect of High-Intensity and Concurrent Training in Body Composition in Costa Rican Overweight and Obese Women
}

\section{Jorge Cervantes-Sanabria* and Jessenia Hernández-Elizondo}

\author{
School of Physical Education and Sports, University of Costa Rica, Costa Rica
}

\begin{abstract}
This study was designed to determine the changes in body composition, Body Weight (BW), Fat Mass (FM), Body Fat Percentage (BF\%) and, Skeletal Muscle Mass (SMM) of High Intensity Interval Training (HIIT) and Concurrent Training (CT), comparing them to a Control Group (CG) for four months of intervention in overweight and obese women.

Methodology: 67 obese and overweight women $(37.54 \pm 8.8$ years, $\mathrm{BMI}=28.49 \pm 4.9, \mathrm{BF} \%=43.63 \pm 5.6 \%)$ were randomly assigned to three groups, HIIT $(\mathrm{n}=26$, mean age $=37.9 \pm 7.3$ years, $\mathrm{BF} \%=42.36 \pm 4.3 \%)$, CT $(\mathrm{n}=20$, mean age $=38.0 \pm 8.5$ years, $\mathrm{BF} \%=45.98 \pm 5.4 \%)$ and $\mathrm{CG}(\mathrm{n}=21$, mean age $=36.6 \pm 11.0$ years, $\mathrm{BF} \%=42.97 \pm 6.8 \%)$. Before and after 16 weeks of HIIT (intervals at $\geq 80 \%$ Maximal Heart Rate) and CT (strength training, 65\%-75\% 1RM + endurance training, 60\%-80\% Maximal Heart Rate), participants underwent Dual-Energy X-Ray Densitometry (DEXA). The CG did not exercise at all.

Results: There were no changes in BW among the experimental groups ( $\mathrm{p}>0.05)$. Compared to CG, the HIIT and CT groups decreased FM, BF\% and increased SMM $(\mathrm{p}<0.05)$. CT was better than HIIT on FM (relative change, $\Delta \%$, HIIT $=$ $-3.4 \%$ vs. $\mathrm{CT}=-4.5 \%), \mathrm{BF} \%(\mathrm{HIIT}=-2.6 \%$ vs. $\mathrm{CT}=-4.3 \%)$ and $\mathrm{SMM}(\mathrm{HIIT}=+1.7 \%$ Vs. $\mathrm{CT}=+5.4 \%)$.

Conclusion: Findings show improvements of body composition in overweight and obese women. In this context, although the results were not highly marked, CT seems to be a better approach for the prevention and management of the women overweight and obesity than HIIT.
\end{abstract}

Keywords

Obese, Overweight, Women, Exercise, High intensity, Concurrent training

Abbreviations

ET: Endurance Training; ANOVA: Analysis of Variance; BF\%: Body Fat Percentage (BF\%); BW: Body Weight; CT: Concurrent Training; CG: Control Group; DEXA: Dual-Energy X-Ray Densitometry; EG; : Experimental Group ; EG $_{2}$ : Experimental Group ${ }_{2}$; FM: Fat Mass; SMM: Skeletal Muscle Mass; HR: Heart Rate; HIIT: High Intensity Interval Training; $\mathrm{HR}_{\max }$ : Maximum Heart Rate; $\mathrm{VO}_{2 \max }$ : Maximum Oxygen Consumption; MET: Metabolic Equivalent of Task; MS: Muscular Strength; R: Randomization

\section{Introduction}

The high prevalence of overweight and obesity in developing countries are a result of continued changes to lifestyle patterns, such as diet and physical activity, leading to an accelerated increase in overweight, obesity, and related chronic diseases in many countries [1]. Today, overweight and obesity remain a major public health concern, and exercise interventions have been pointed out as an important target in the prevention of weight gain and overweight/obesity development [2].

It has showed that 8 months of Strength Training (ST), (3 times per week, 3 sets, 8 -12 repetitions), Endurance Training (ET), (12 miles per week at $65-80 \%$ peak $\mathrm{VO}_{2}$, performed on a treadmill, elliptical trainers or cy- cle-ergometer) or their combination can improve the body composition variables of overweight/obese adults [3]. To perform ST 2-3 times a week, in combination with ET is recommended for these individuals to maintain or improve their health $[4,5]$.

*Corresponding author: Jorge Cervantes Sanabria, Schoo of Physical Education and Sports, University of Costa Rica, Costa Rica, E-mail: jcersa3@yahoo.es

Received: June 27, 2017: Accepted: September 23, 2017: Published online: September 25, 2017

Citation: Cervantes J, Hernández J (2017) Effect of High-Intensity and Concurrent Training in Body Composition in Costa Rican Overweight and Obese Women. Arch Sports Med 1(2):65-74

Copyright: (C) 2017 Sanabria JC, et al. This is an open-access article distributed under the terms of the Creative Commons Attribution License, which permits unrestricted use, distribution, and reproduction in any medium, provided the original author and source are credited. 
An excess of body fat has been identified as one of the most important risk factors for chronic disease morbidity and mortality in men and women. Most women and men, gain fat through middle adulthood and adult weight gain has many adverse health implications: the risk of heart disease, diabetes, and certain cancers is substantially elevated with higher weight [6]. Women experience a reduction in basal metabolic rate and loss of Skeletal Muscle Mass (SMM) as they transition to menopause; thereby increasing their risk for body weight gain and obesity [7].

High Intensity Interval Training (HIIT) promotes positive adaptations that attenuate the effects of obesity [8] and could be used as a time-effective therapy for the management of body fat levels in overweight and obese women [9]. HIIT has demonstrated significant reductions $(\mathrm{p}<0.05)$ in body composition variables such as Body Weight (BW), Fat Mass (FM) and Body Fat percentage (BF\%) in women [10-12]. For example, Trapp, et al. [10] carried out a 15 weeks of HIIT intervention (20 min per session, 60 repetitions, $8 \mathrm{~s}$ maximal effort intervals with $12 \mathrm{~s}$ rest, 3 times per week performed on a cycle-ergometer) in obese young women $(20.2 \pm 2.0$ years old) and their results shows significant reductions in $\mathrm{FM}(\Delta \%=-11.11 \%)$ and $\mathrm{BF} \%(\Delta \%=-7.69 \%)$ without significant changes in SMM ( $p>0.05)$. The key of HIIT prescription is training intensity; it is characterized by a physical effort of $80 \%$ to $100 \%$ of maximum Heart Rate $\left(\mathrm{HR}_{\max }\right)$ in intermittent interval training sessions of up to 30 minutes [13].

On the other hand, over recent years, a growing body of scientific knowledge strongly suggests that regular performance of ST and ET is a major factor in the prevention and treatment of cardiovascular disease including risk factors such as obesity [14]. Concurrent training (CT, ST+ET) has demonstrated results in loss of fat mass percentage [15-17] and important increments in SMM [18].

Previous studies have combined HIIT and ST, showing improvements in health parameters like body composition and it has previously been shown that, during CT, body fat decreased more with increasing ET intensities [19]. Including exercise modes into the same training regimen may be even more effective than adhering to only either training mode alone for this target [20].

However, there is currently a dearth of knowledge about the independent effects of HIIT and CT on body composition, relative to the effects of others exercise modes [21]. For example, Trilk, et al. [22] applied a HIIT protocol in a period of 4 weeks (4-7 intervals of $30 \mathrm{~s}$ with 4 min rest, 3 times per week performed on a cycle-ergometer) and they did not meet significant changes in BW, FM, BF\% ( $\mathrm{p}>0.05)$ of overweight women (30.1 \pm 6.8 years old) tested by DXA, and Ali-Mohamadi, et al.
[23] carried out a CT intervention for 8 weeks in overweight women $(23.2 \pm 2.4$ years old) who performed a ST program (6 exercises, 3 sets of 8 repetitions at $80 \% 1 \mathrm{RM}$, 2-3 min rest between sets and 3-5 min between each exercise) and ET program (10 min +30 s per session at 75 $\left.80 \% \mathrm{HR}_{\max }\right) 3$ times per week. They obtained significant reductions $(\mathrm{p}<0.05)$ in FM $(\Delta=-26.95 \%)$ and $\mathrm{BF} \%(\Delta=$ $-23.23 \%)$, but not in SMM ( $p>0.05)$.

Due to the difference in procedures among them, most of the training protocols show dissimilarities in variables such as frequency, intensity, duration and type of exercise, which make it more difficult to understand which methods are the most beneficial to overweight and obese women.

HIIT clearly provides a potent physiological stimulus for improving body composition, but, there are many questions that remain. It has been widely accepted that SMM is important for regulating multiple metabolic processes, therefore it is critical to identify modes of exercise training that can result in loss of fat mass while also preserving SMM or even building SMM in a more effective way and HIIT could provide a potent anabolic response for overweight and obese women [24].

Therefore, this study aimed to determine the changes in body composition based on two training programs, HIIT and CT, by comparing them with a Control Group (CG) over the course of four months in overweight and obese women. Session duration in both training programs was compared by taking into account the caloric expenditure (METs) so as to minimize any imbalance in physical effort protocols.

\section{Methods}

The study was performed over a period of 16 weeks. Participants were randomly separated into 3 groups. Group 1 performed 16 weeks of HIIT, group 2 performed $\mathrm{CT}$ and group 3 was asked to continue with their routines and not to engage in any physical activity during the research period.

\section{Subjects}

Participants were recruited through Facebook and poster advertisements. All participants completed health history and physical activity questionnaires and met the following inclusion criteria: 18-years-old or older, physically independent, body fat percentage $\geq 26 \%$ as overweight and obesity indicator [25], free from cardiac or orthopedic problems, not receiving nutritional therapy, not smokers, and not be pregnant before start of the study. One hundred four $(\mathrm{n}=104)$ women were accessed for eligibility. After individual interviews, 28 women who did not meet the inclusion criteria were excluded. The remaining 89 women were selected for par- 
ticipation, then were randomly assigned to 1 of 3 groups: Group 1 (HIIT) performed the HIIT protocol $(\mathrm{n}=31)$, Group 2 (CT) performed the CT protocol $(n=29)$ or a Control Group (CG) in the first phase of the study protocol. A total of 67 participants $(37.54 \pm 8.84$ years; 1.59 $\pm 0.68 \mathrm{~m}, \mathrm{BF} \%=43.63 \pm 5.6 \%$ ) completed all stages of the study and were included in the analyses. The reasons for withdrawal from the study were reported as lack of time, transportation issues, lack of motivation, personal reasons and, absences $\geq 10 \%$. Adherence to the program was satisfactory. The rate of attendance was $93.91 \%$ for the HIIT, $95.1 \%$ for the CT and $72.4 \%$ for CG over the course of the entire research project. None of the participants suffered any injury during this period of time.

Written informed consent was obtained from all subjects after a detailed description of study procedures was provided. This investigation was conducted according to the Declaration of Helsinki and was approved in Session 293, VI-2964-2015 by the Scientific Ethics Committee of the University of Costa Rica in May of 2015.

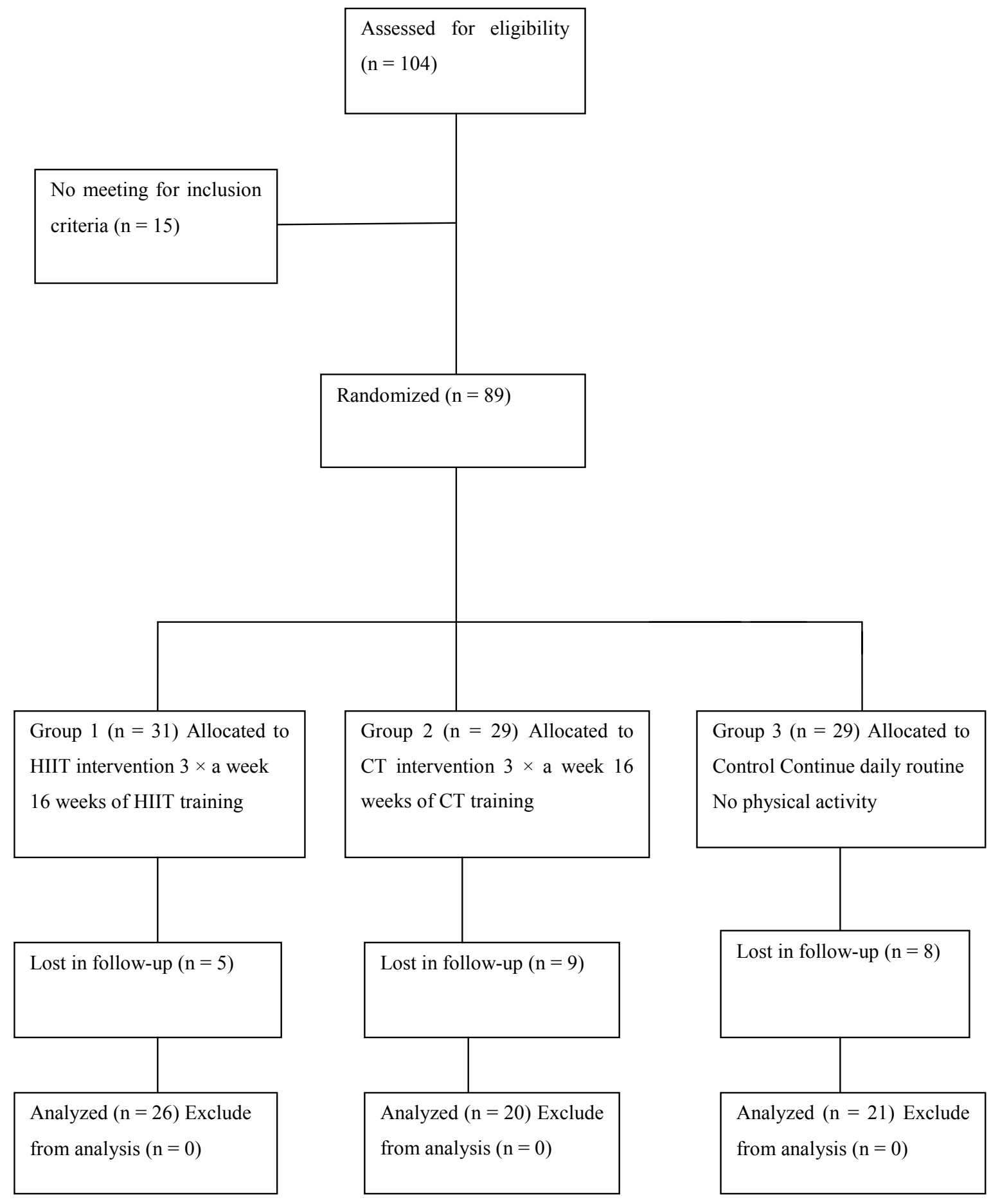

Figure 1: Experimental design. 


\section{Procedures}

At the beginning and the end of the experiment, 2 weeks were allocated for evaluations (weeks 1-16), consisting of body composition analysis by Dual-Energy X-Ray Absorptiometry (DXA). This was done at the Body Composition Laboratory at Research Center in Sciences of Human Movement (CIMOHU, for its acronym in Spanish), University of Costa Rica. The experimental design is displayed in Figure 1.

\section{Body composition}

Changes in body composition variables BW, FM, BF\% and SMM were measured by Dual-Energy X-Ray Densitometry (DEXA) with a densitometry model Lunar Prodigy Advanced PA+350411 of GE Healthcare with a $2 \%$ margin of error. A complete body exam was applied to every subject at the beginning and end of study. Before the test, participants were instructed to lie on a flat table face up and remain still during the scan.

SMM was estimated by the predictive equation proposed by Kim, et al. [26] and it was determinate as FatFree Muscle Mass (FFMM).

\section{Muscle strength}

One repetition maximal was evaluated for maximal dynamic strength in exercises chest press, leg press and biceps curl only for Strength Training (ST) exercise prescription. A warm-up set (6-10 repetitions) with light load to familiarize the subjects with the testing equipment and lifting technique was preceded for each exercise testing. Procedure was initiated 2 minutes after the warm-up set and subjects were instructed to try to accomplish 2 repetitions with the load in 3 attempts in all exercises tested. A period of 3-5 minutes rest was given between each attempt and 5 minutes between exercises. The last resistance lifted in which the subject was able to complete only 1 repetition was recorded. Each exercise technique was standardized and continuously monitored to ensure reliability. 1RM testing sessions were supervised by one experienced researcher to maximize safety and test reliability. Verbal encouragement was given throughout each test [27].

\section{Training Programs}

\section{Concurrent training}

Supervised CT was performed in the Fitness Gym facilities in Cartago, Turrialba. (Cartago is a Costa Rican province and Turrialba is a city in that province). The protocol was based on recommendations for CT $(\mathrm{ST}+\mathrm{ET})$ in overweight and obese women population to improve body composition [4] and, it was made in a novel way. All participants were personally supervised by a physical education professional to help ensure consistent and safe exercise performance.

\section{Strength training program}

Subjects performed ST using a combination of free weights and machines. The sessions were performed 3 times per week in non-consecutive days. The ST program was a whole-body program of 8-9 exercises (Table 1) with 4-5 exercises performed with free weights and 4 with machines. Exercises were performed in the following order: Upper-limb, lower limb and, core. Some exercises were changed every 4 weeks to maintain the neuromuscular stimulus of the participants.

Participants performed 2-4 sets of 15-8 repetitions with a load of $65-75 \%$ of $1 \mathrm{RM}$. Participants were instructed to inhale during the eccentric phase and exhale during the concentric phase of each repetition while maintaining a constant velocity of movement at a ratio of approximately 2:2 (concentric and eccentric phases, respectively). Participants were allowed 1-2 minutes of rest between sets of an exercise and 2-3 minutes between exercises.

The loads of each exercise were adjusted according to the improvements in exercise capacity for 1RM each 4 weeks. The subjects were exercising with as much resistance as possible while maintaining proper exercise technique throughout the study. Progression for the ST was accomplished by increasing the load for $5 \%$ of $1 \mathrm{RM}$ each 4 weeks.

\section{Endurance training program}

In the ET sessions the subjects underwent a heart rate-controlled running training program with cardiac monitors for beginners outdoors. During the first 2 weeks, participants alternate between walking and running in order to allow them to get used to the training load; after 3 weeks, the subjects ran, if possible, continuously at an intensity of $70-80 \%$ of their maximal heart rate as determined by the formula 220-age [4] for 20-30 min through the study.

CT participants did 20 minutes of ST and 20 minutes of ET during the first weeks of the research period and 30 minutes of ST and 30 minutes of ET during the last weeks of the research period. All of the participants did 5 minutes of warm-up and 5 minutes of stretching before each session and 5 additional minutes of stretching at the end of each session. The caloric expenditure was approximately 3.62 METs in the first sessions and, 4.54 METs for last sessions [28]. The session's duration was $40 \mathrm{~min}$ in weeks 1-8 and $60 \mathrm{~min}$ in weeks 9-16. Protocol of CT prescription is displayed in Table 1.

\section{High intensity interval training program}

Subjects performed HIIT using their body weight. The sessions were performed 3 times per week in non-con- 
secutive days. The HIIT program was a whole-body program of 10 body exercises in the first 8 weeks with a HIIT commercial video called Insanity Max30 Program ${ }^{\circledR}$ in the last 8 weeks of the study. Exercises were performed in the following order: Jogging, jumping jacks, high knees, 1-2-3 drills, push-ups, frog jumps, squats, plank touches, jump rope, 1-2-3 knees up. Some exercises were changed every 3 weeks to maintain the neuromuscular stimulus of the participants.

Participants performed 10-12 intervals of 30-45 seconds with their body weight load for 8 weeks and the last 8 weeks performed 6 intervals of 5 min with Insanity Max30 Program ${ }^{\circledR}$. Participants were instructed to maintain a constant velocity of movement according to their physical capacity. Participants were allowed $1 \mathrm{~min} 30 \mathrm{~s}$ to of rest between intervals at the beginning of the study and $30 \mathrm{~s}$ at the end.

Progression for HIIT was adjusted by raising the number of intervals and time of exercise and, reducing time of rest. The subjects were exercising with as much resistance as possible while maintaining proper exercise technique throughout the study. All of the participants did 5 minutes of warm-up and 5 minutes of stretching before each session and 5 additional minutes of stretching at the end of each session. The duration of the HIIT sessions was 35-45 minutes long with a caloric expenditure similar of CT [28]. All participants were personally supervised by a physical education professional to help ensure consistent and safe exercise performance. Protocol of HIIT prescription is displayed in Table 2.

\section{Statistical Analyses}

Analyses of Variance (ANOVA) in two-factor mixeddesign ANOVA with repetitive measures in one factor (3 groups $\times 2$ measures) was applied with SPSS statistical program version 21.0. In addition, statistical monitoring was applied to the significant interactions using a post hoc analysis of simple effects to determine the differences between groups and measurements when needed. The

Table 1: Summary of the concurrent training programmes (strength training follow to endurance training).

\begin{tabular}{|c|c|}
\hline & ST + ET \\
\hline \multirow[t]{2}{*}{ Warm-up period } & 5 min of jogging at low intensity \\
\hline & 5 min of dynamic stretching exercises \\
\hline \multirow{5}{*}{$\begin{array}{l}\text { Concurrent } \\
\text { training period }\end{array}$} & Weeks 1-2: 6 sessions, pre-test and familiarization \\
\hline & $\begin{array}{l}\text { Weeks 3-4: ST: } 2 \text { sets } \times 12 \text { reps at } 65 \% 1 \mathrm{RM}, 1 \text { min rest, } 8 \text { exercises (UL: chest press, front pull, biceps curl. } \\
\text { LL: squat, leg press, knee extension, knee flexion. C: } 30 \text { s plank) }+\mathrm{ET}: 20 \text { min running/walking at } 60 \% \mathrm{HR}_{\text {man }}\end{array}$ \\
\hline & $\begin{array}{l}\text { Weeks 5-8: ST: } 12 \text { sessions, } 4 \text { sets } \times 10 \text { reps at } 70 \% 1 \mathrm{RM}, 1 \text { min rest, } 9 \text { exercises (UL: inclined chest press, } \\
\text { chest fly, military press, biceps curl. LL: sumo squat, leg press, knee extension, knee flexion, calf raise. C: } 30 \\
\text { s plank) }+20 \text { min running at } 65 \% \mathrm{HR}_{\text {max }}\end{array}$ \\
\hline & $\begin{array}{l}\text { Weeks 9-12: ST: } 12 \text { sessions, } 4 \text { sets } \times 8 \text { reps at } 75 \% 1 \mathrm{RM}, 2 \text { min rest, } 10 \text { exercises (UL: inclined chest press, } \\
\text { chest fly, military press, biceps curl. LL: sumo squat, leg press, knee extension, knee flexion, calf raise. C: } 30 \\
\text { s high to low plank) + ET: } 30 \text { min running at } 70 \% \mathrm{HR}_{\max }\end{array}$ \\
\hline & $\begin{array}{l}\text { Weeks 13-16: ST: } 12 \text { sessions, } 4 \text { sets } \times 8 \text { reps at } 75 \% 1 \mathrm{RM}, 2 \text { min rest, } 10 \text { exercises (UL: chest press, peck } \\
\text { deck, face-pull, Arnold press, biceps curl. LL: leg press, lunges, knee extension, calf raise. C: } 30 \mathrm{~s} \text { high to low } \\
\text { plank) + ET: } 30 \text { min running at } 75 \% \mathrm{HR}_{\max } \text {, post-test }\end{array}$ \\
\hline $\begin{array}{l}\text { Cool down } \\
\text { period }\end{array}$ & 5 min of static stretching exercises \\
\hline
\end{tabular}

UL: Upper Limb; LL: Lower Limb; C: Core.

Table 2: Summary of the high intensity interval training programmes.

\begin{tabular}{|c|c|}
\hline & HIIT \\
\hline \multirow[t]{2}{*}{ Warm-up period } & 5 min of jogging at low intensity \\
\hline & 5 min of dynamic stretching exercises \\
\hline \multirow{5}{*}{$\begin{array}{l}\text { Interval training } \\
\text { period }\end{array}$} & Weeks 1-2: 6 sessions, pre-test and familiarization \\
\hline & $\begin{array}{l}\text { Weeks 3-4: } 20 \text { min, } 10 \text { intervals of } 30 \mathrm{~s} \text { at }>80 \% \mathrm{HR}_{\max }, 1 \mathrm{~min} 30 \mathrm{~s} \text { rest between intervals, } 10 \text { exercises } \\
\text { (Jogging, jumping jacks, high knees, } 1-2-3 \text { drills, push-ups, frog jumps, squats, plank touches, jump rope, } \\
\text { 1-2-3 knees up) }\end{array}$ \\
\hline & $\begin{array}{l}\text { Weeks 5-8: } 20 \text { min, } 12 \text { intervals of } 45 \mathrm{~s} \text { at }>80 \% \mathrm{HR}_{\max }, 1 \text { min rest between intervals, } 10 \text { exercises (Jogging, } \\
\text { cross jumping jacks, high knees with punches, in - out abs, push-ups, frog jumps, squats jumps, plank } \\
\text { touches, jump rope, } 1-2-3 \text { knees up) }\end{array}$ \\
\hline & $\begin{array}{l}\text { Weeks 9-12: } 30 \text { min, } 6 \text { intervals of } 5 \text { min at }>80 \% \mathrm{HR}_{\text {max }}, 30 \text { s rest between intervals, Insanity Max } 30^{\circledR} \\
\text { Program (weeks 9-10: Cardio Challenge Video, weeks } 11-12 \text { : Sweat Intervals Video) }\end{array}$ \\
\hline & $\begin{array}{l}\text { Weeks 13-16: } 30 \mathrm{~min}, 6 \text { intervals of } 5 \mathrm{~min} \text { at }>80 \% \mathrm{HR}_{\max }, 30 \mathrm{~s} \text { rest between intervals, Insanity Max } 30^{\circledR} \\
\text { Program (weeks 13-14: Max Out Cardio Video, weeks 15-16: Max Out Sweat Video), post-test }\end{array}$ \\
\hline Cool down period & 5 min of static stretching exercises \\
\hline
\end{tabular}




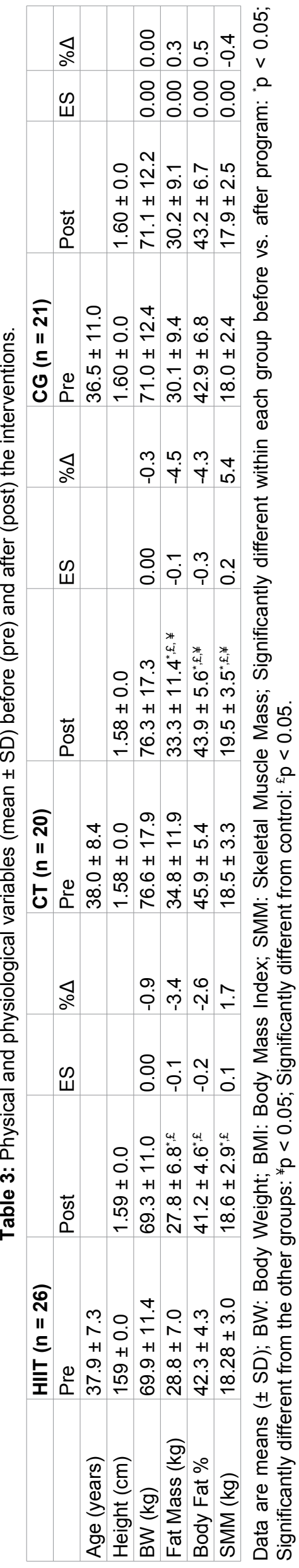

significance level of $95 \%(p<0.05)$ was useful for every case. Both Hedges'g effect size [29] and the corresponding percentage changes were applied. To calculate Hedges' $g$ size effect size [29], the following formula was used:

$$
\begin{aligned}
& g=\frac{\text { Posttest }- \text { Pretest }}{D S} \\
& D S=\sqrt{\frac{\left(n_{\text {post }}-1\right) D S_{p o s t^{2}}+\left(n_{\text {pre }}-1\right) D S_{p r e^{2}}}{n_{\text {post }}+n_{\text {pre }}-2}}
\end{aligned}
$$

$\mathrm{n}=$ number of subjects.

Based on Thomas, Salazar \& Landers [30]. Size Effect (ES) is interpreted as $<0.40$ small, $>0.40 \mathrm{a}<0.70$ moderate, $>0.70 \mathrm{big}$. Changing percentage was calculated using the following formula:

$$
\Delta \%=\frac{\text { Posttest }- \text { Pretest }}{\text { Pretest }} * 100
$$

\section{Results}

Forty-six $(\mathrm{n}=46)$ overweight and obese women completed a total of 48 training sessions and 67 of them applied both pre-tests and post-tests. The sample was composed for $44 \%$ housewives, $40 \%$ workers and, $14 \%$ university students. The physical and physiological variables of the three groups at the beginning and at the end of the 16week period are summarized in Table 3 . The probability of a Type II Error was 78\% for FM, 94\% for BF\% and 99\% for SMM. Comparisons of the three groups (HIIT, CT and $\mathrm{CG}$ ) showed that they were homogenous for body composition parameters, BW ( $p=0.256), F M(p=0.970), B F \%$ $(\mathrm{p}=0.820)$ and, SMM $(\mathrm{p}=0.863)$ before the commencement of the training program. At the post-intervention, a not significant change $(p>0.05)$ in the BW of all groups was obtained. On the contrary, FM decreased significantly in HIIT group $(\mathrm{p}<0.05, \mathrm{ES}=-0.14)$ and in CT group $(\mathrm{p}$ $<0.05, \mathrm{ES}=-0.13)$. In the between groups' comparison, FM in CT group were significantly different $(\mathrm{p}<0.05)$ from the two other groups (HIIT and CG). Similarly, in post-intervention, the HIIT participants' BF\% decreased significantly $(\mathrm{p}<0.05, \mathrm{ES}=-0.24)$. In $\mathrm{CT}$ group, the $\mathrm{BF} \%$ was also significantly decreased ( $p<0.05, E S=-0.36)$. In the between groups' comparison, $\mathrm{BF} \%$ in $\mathrm{CT}$ group were significantly different $(\mathrm{p}<0.05)$ from the two other groups (HIIT and CG). At the end of the training period, a significant increase was observed in SMM of both trained groups $(\mathrm{p}<0.05, \mathrm{ES}=0.10$ and 0.29 , for HIIT and CT, respectively), but a higher increase was noted in CT group when compared to HIIT or control groups $(\mathrm{p}<0.05)$.

\section{Discussion}

The main focus of this study was to compare the effects of HIIT versus CT on body composition parameters in overweight and obese women. The present study 
results demonstrated for the first time that both types of training were effective in improving body composition in this sample. A major finding from our study was the trainings groups participants' relatively improvement in BF\%, which was attained through concurrent FM loss and SMM gain (Figure 2). No changes were observed in BW for both training groups and for all variables in CG.

Exercise alone is not an effective method for achiev- ing initial weight loss. Without calorie restriction, weight loss through exercise alone is quite small, about $0.1 \mathrm{~kg} /$ week after 12 months, but, even in the absence of significant weight loss, regular aerobic and resistance exercise improves cardiovascular fitness and obesity-related comorbidities [31]. According to the American College of Sports Medicine's position, ST and ET combined are superior to either exercise modality alone with respect to
A)

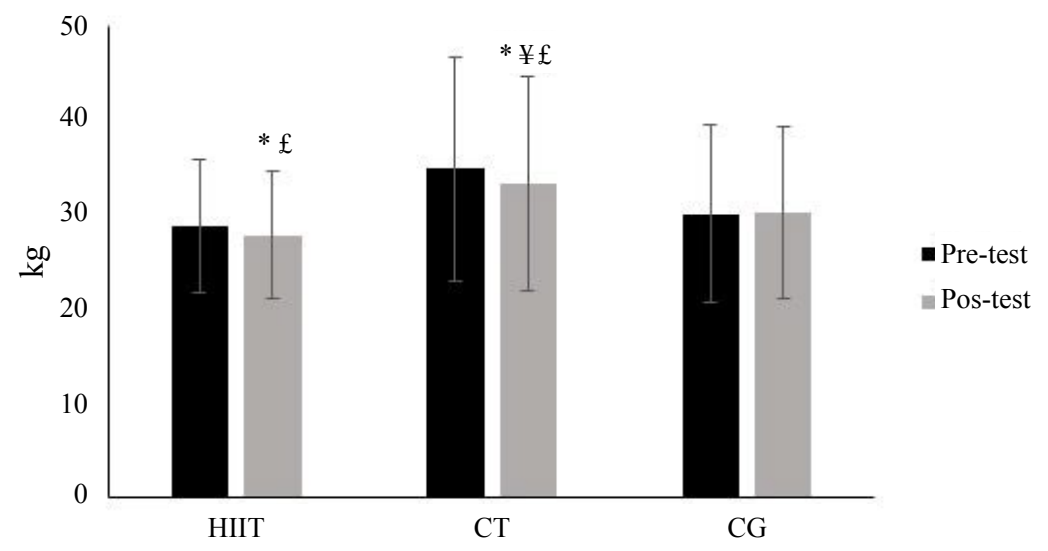

B)

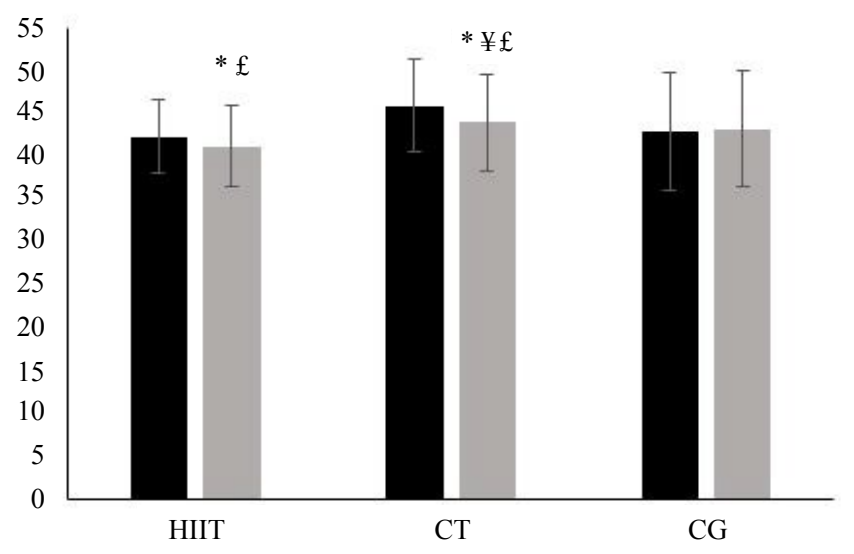

C)

SMM

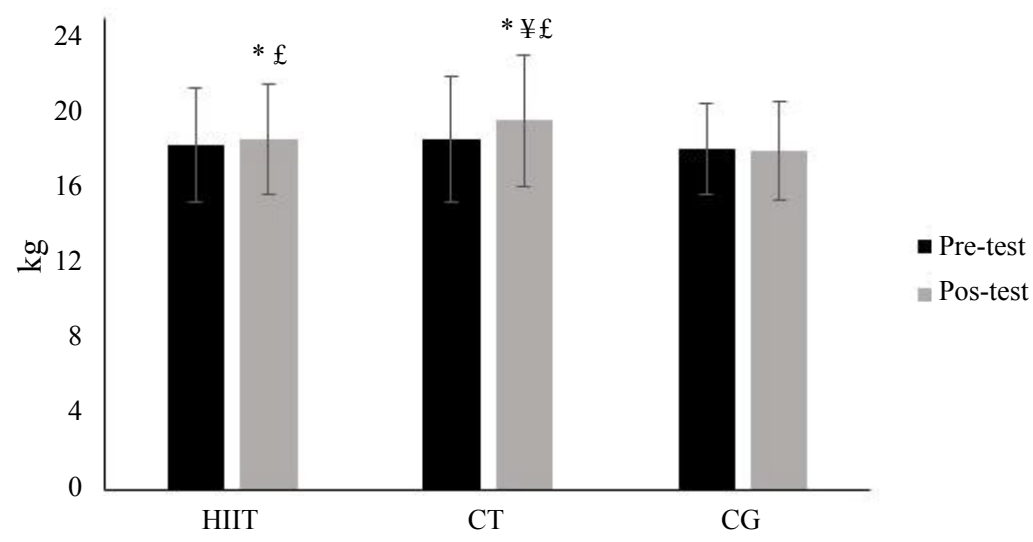

Figure 2: Fat mass, body fat percentage, and skeletal muscle mass at pre- and post-training. " $p<0.05$ pre-training vs. posttraining, ${ }^{£} p<0.05$ training groups vs. control, ${ }^{\sharp} p<0.05$ HIIT vs. CT. Data are expressed as mean and SD. 
concurrent FM loss and SMM gain. Based on our participants' significant improvements in body composition, the combined training approach appears to be a more effective intervention for overweight and obese women than performing HIIT alone [32].

In our study, three sessions per week of HIIT and CT during 16-week period provided a physiological stimulus for improving body composition. We identify two potential modes of exercise training prescription that can result in FM loss while also preserving SMM or even building SMM, in four month. Fisher, et al. [24] proposed that HIIT could provide a potent anabolic response for overweight and obese women SMM, and in our study, this statement was observed briefly. This is a preliminary result that needs to be more investigated.

Previous studies with similar intervention periods have demonstrated the benefits of HIIT and CT in women body composition, but, some aspects are not clear in overweight and obese women yet. Respect to HIIT, Trapp, et al. [10] carried out a 15 weeks of HIIT intervention $(20 \mathrm{~min}$ per session, 60 repetitions, $8 \mathrm{~s}$ maximal effort intervals with $12 \mathrm{~s}$ rest, 3 times per week performed on a cycle-ergometer) in obese young women (20.2 \pm 2.0 years old) and their results shows significant reductions in $\mathrm{FM}(\Delta \%=-11.11 \%)$ and $\mathrm{BF} \%(\Delta \%=-7.69 \%)$ without significant changes in SMM ( $\mathrm{p}>0.05)$. Comparing our results with Trapp, et al. [10], a protocol based on cycle-ergometer is better to decrease FM and BF\%, but not to improve SMM. In this context, our protocol intervention shows be potentially more effective than cycle-ergometer HIIT for building SMM, and less effective, but similarly beneficial to decrease FM and BF\% tested by DXA. Of course, this relationship should be analyzed in a forward experimental study.

Shorter periods of HIIT should appear being less effective to improve body composition in overweight women. Trilk, et al. [22] applied a HIIT protocol in a period of 4 weeks (4-7 intervals of $30 \mathrm{~s}$ with 4 min rest, 3 times per week performed on a cycle-ergometer) and they did not meet significant changes in BW, FM, BF\% ( $p$ $>0.05)$ of overweight women $(30.1 \pm 6.8$ years old) tested by DXA. In contrast, Kordi, et al. [11] in a HIIT period of 6 weeks met significant decreases in BF\% (-11.78\%) tested by skinfolds in obese young women $(18.64 \pm 0.6$ years old), while Airin, et al. [12], at the same period exercise, obtained significant changes in BW (-1.9\%), BF\% $(-2.2 \%)$ and SMM $(+1.15 \%)$ by bio-impedance in overweight women $(20.5 \pm 1.9$ years old $)$.

In the Trapp, et al. [10] study and our study, the results were not as high as those obtained by Kordi, et al. [11] in BF\% and both contrast with the results met by Trilk, et al. [22] tested by DXA. According to this con- text, the training duration of HIIT and the instrumental used to measure body composition variables could be fundamental to explain the role of HIIT in overweight and obese women. The changes in body composition reported by shorter studies applying skinfolds and bio-impedance how its measure's instrument have to be carefully analyzed.

Respect to CT, Ali-Mohamadi, et al. [23] carried out a CT intervention for 8 weeks in overweight women $(23.2$ \pm 2.4 -years-old). They performed a ST program (6 exercises, 3 sets of 8 repetitions at $80 \% 1 \mathrm{RM}, 2-3$ min rest between sets and 3-5 min between each exercise) and ET program $\left(10 \mathrm{~min}+30 \mathrm{~s}\right.$ per session at $\left.75-80 \% \mathrm{HR}_{\max }\right) 3$ times per week. The results of this study show significant reductions $(\mathrm{p}<0.05)$ in $\mathrm{FM}(\Delta=-26.95 \%)$ and $\mathrm{BF} \%(\Delta$ $=-23.23 \%)$, but not in SMM ( $\mathrm{p}>0.05)$. Compared to our study, the ST program (8-9 exercises, $2-4$ sets of $15-8$ repetitions at $65-75 \% 1 \mathrm{RM}, 1-2$ between sets and 2-3 min between each exercise) and the ET program (20-30 min per session at $70-80 \% \mathrm{HR}_{\max }$ ) were more physically demanding for a longer duration and overweight women participants presented values in body composition (BW $=73.7 \pm 9 \mathrm{~kg}, \mathrm{FM}=17.7 \pm 2.9 \mathrm{~kg}, \mathrm{BF} \%=24 \pm 2.3)$ similar to our study $(\mathrm{BW}=76.6 \pm 17.9, \mathrm{FM}=34.4 \pm 11.9 \mathrm{~kg}$ and, $\mathrm{BF} \%=45.9 \pm 5.4$ ), but, our results appear to be less effective than this research.

Due to these similarities in exercise protocol, the body composition characteristics of the sample and the shorter duration of the intervention, the lack of effectiveness observed in our results could be related to the instrument application for these authors in body composition. Skinfolds measurements method seems to include a systematic error in the fat analysis because of its high vulnerability to external factors such as researcher experience or instrument calibration [33] that demand to analyze conclusions using this technique with caution.

The results reported in this study were similar to those reported by Trapp, et al. [10] where intermittent exercises were appropriate and was observed to provide significant improvements in BM and BF. In contrast, when the exercise modality was different, based on cycle-ergometer, the same author Trapp, et al. [10] reported contradictory results in the SMM of obese women compared with our study. Others authors [22] not reported significant results when the exercise modality was different in time of intervention and some were confused when the difference was the instrument employed to measure body composition [11,12,23].

Physiologically, the improvement of both training groups in FM and BF\% could be explain by the role of post-exercise lipolysis and energy expenditure for demanding and specific exercise. HIIT and CT require glucose catabolism and in consequence the glucose metabolism is turned away to restore mus- 
cle glycogen stocks. In the post-exercise recovery period, muscle glycogen resynthesis is the high metabolic priority, inducing post-exercise lipid combustion [8]. Although post-exercise lipolysis works differently in each of the exercising groups for this research, both training methods have demonstrated similar 24-hour energy expenditure in the post-exercise time [34].

In regards to the SMM, the continue training sessions suggest important metabolic demands, such as endogenous reserve exhaustion (hepatic glycogen and muscular), body fluid and electrocute, hormonal imbalance, and/or imbalance in muscular proteins $[19,35]$ that could originate a chronic muscular hypertrophy due to the increment in the muscular fiber, more actin and myosin, sarcoplasmic, connective tissue or any combination of them if the stimulus keeps constant [36].

Some limitations should be mentioned in this research study design. In order to reduce a potential drop out of the study, and to take account some recommendations in previous experiences, we did not control feeding habits neither tested the follicular stage of the menstrual cycle in overweight and obese women because we did not want to put the living habits of the participants under much stress. This strategy was insufficient to prevent drop-out, therefore, we investigated a small sample of overweight and obese women $(n=67)$.

Another important aspect of this study was the adherence to exercise despite of drop-out. It is noted that 9 subjects were eliminated from CT for failure to complete the required training sessions, while only 5 were eliminated form HIIT. The time session was important according to some explanations of the participants. Protocols such as HIIT has to be considered in designing training protocols for overweight and obese women, because of it probably contributes to lower drop-out rates compared to CT. Nevertheless, given the extreme nature of the exercise protocol, it may be doubtful that the general population could safely or practically adopt the model of HIIT and some displeasure could occur for the overweight/obese adults and some other clinical populations [8].

\section{Conclusion}

The most important findings of the present study were the improvements of body composition in overweight and obese women. In this context, although the results were not highly marked, CT seems to be a better approach for the prevention and management of the women overweight and obesity than HIIT. However, the decision on the type of exercise prescription should be individualized to optimize health status variables' improvement and to enjoy the exercise.

\section{Acknowledgments}

The present study was supported by the University of Costa Rica, Faculty of Education, School of Physical Edu- cation and Sports, Master Degree Program. The authors are grateful to all of the women for their cooperation; we also thank Dr. Jose Moncada Jimenez, Dr. Andrea Solera Herrera, M.Sc. Isaura Castillo Hernández, M.Sc. Yamileth Chacón Araya and Bach. Damián Chavarría for their assistance.

\section{Institutional Revision}

This study was designed based on the participants' rights of the National Health Board (Consejo Nacional de Salud, CONIS, for its acronym in Spanish) of the Costa Rican Ministry of Health and it was ensured by the Ethical Scientific Committee of the Vicerrectoría de Investigación of the University of Costa Rica (Session No. 293, VI-2964-2015).

All procedures were fully explained to the participants who signed a written informed consent form meeting the principles of the Ethical Scientific Committee of the Vicerrectoría de Investigación of the University of Costa Rica (Session No. 293, VI-2964-2015).

\section{References}

1. Doak CM, Popkin BM (2017) Overweight and obesity. Nutrition and Health in a Developing World. Springer International Publishing, 143-158.

2. Rautiainen S, Wang L, Lee IM, et al. (2016) Dairy consumption in association with weight change and risk of becoming overweight or obese in middle-aged and older women: A prospective cohort study. Am J Clin Nutr 103: 979-988.

3. Willis LH, Slentz CA, Bateman LA, et al. (2012) Effects of aerobic and/or resistance training on body mass and fat mass in overweight or obese adults. J Appl Physiol (1985) 113: $1831-1837$

4. (2014) ACSM's guidelines for exercise testing and prescription/American College of Sports Medicine. In: Senior, Linda S Pescatello, Ross Arena, Deborah Riebe, Paul D. Thompson. (9 $9^{\text {th }}$ edn).

5. Rustaden AM, Haasktad LA, Paulsen G, et al. (2017) Effects of BodyPump and resistance training with and without a personal trainer on muscle strength and body composition in overweight and obese women-A randomized controlled trial. Obes Res Clin Pract.

6. Hruby A, Manson JE, Qi L, et al. (2016) Determinants and consequences of obesity. Am J Public Health 106: 1656-1662.

7. McArthur D, Dumas A, Woodend K, et al. (2014) Factors influencing adherence to regular exercise in middle-aged women: A qualitative study to inform clinical practice. BMC Womens Health 14: 49.

8. Racil G, Coquart JB, Elmontassar W, et al. (2016) Greater effects of high-compared with moderate-intensity interval training on cardio-metabolic variables, blood leptin concentration and ratings of perceived exertion in obese adolescent females. Biol Sport 33: 145-152.

9. Keating SE, Machan EA, O'Connor HT, et al. (2014) Continuous exercise but not high intensity interval training improves fat distribution in overweight adults. J Obes 2014: 834865.

10. Trapp E, Chisholm D, Freund J, et al. (2008) The effects of high-intensity intermittent exercise training on fat loss and 
Citation: Cervantes J, Hernández J (2017) Effect of High-Intensity and Concurrent Training in Body Composition in Costa Rican Overweight and Obese Women. Arch Sports Med 1(2):65-74

fasting insulin levels of young women. Int $\mathrm{J}$ Obes (Lond) 32: 684-691.

11. Kordi M, Choopani S, Hemmatinafar M, et al. (2013) The effects of the six week high intensity interval training (HIIT) on resting plasma levels of adiponectin and fat loss in sedentary young women. Journal of Jahrom University of Medical Sciences 11: 23-31.

12. Airin S, Linoby A, Zaki M, et al. (2014) The Effects of High-Intensity Interval Training and Continuous Training on Weight Loss and Body Composition in Overweight Females. Proceedings of the International Colloquium on Sports Science. Exercise, Engineering and Technology 2014, 401-409.

13. McGlinchy S (2012) The Effect of Two High Intensity Interval Training Protocols on Heart Rate, Caloric Expenditure, and Substrate Utilization During Exercise and Recovery. University of Toledo. Theses and Dissertations, 374.

14. Schumann M, Küüsmaa M, Newton RU, et al. (2014) Fitness and lean mass increases during combined training independent of loading order. Med Sci Sports Exerc 46: 1758-1768.

15. Dolezal BA, Potteiger JA (1998) Concurrent resistance and endurance training influence basal metabolic rate in nondieting individuals. J Appl Physiol 85: 695-700.

16. Takeshima N, Rogers M, Islam M, et al. (2004) Effect of concurrent aerobic and resistance circuit exercise training on fitness in older adults. Eur J Appl Physiol 93: 173-182.

17. Häkkinen A, Hannonen P, Nyman K, et al. (2003) Effects of concurrent strength and endurance training in women with early or longstanding rheumatoid arthritis: comparison with healthy subjects. Arthritis Rheum 49: 789-797.

18. Arazi H, Faraji H, Moghadam M, et al. (2011) Effects of concurrent exercise protocols on strength, aerobic power, flexibility and body composition. Kinesiology 43: 155-162.

19. Gentil P, de Lira CAB, Cardoso Filho SG, et al. (2017) High intensity interval training does not impair strength gains in response to resistance training in premenopausal women. Eur J Appl Physiol 117: 1257-1265.

20. Eklund D, Häkkinen A, Laukkanen JA, et al. (2016) Fitness, body composition and blood lipids following 3 concurrent strength and endurance training modes. Appl Physiol Nutr Metab 41: 767-774.

21. Decker ES, Ekkekakis $P$ (2017) More efficient, perhaps, but at what price? Pleasure and enjoyment responses to high-intensity interval exercise in low-active women with obesity. Psychology of Sport and Exercise 28: 1-10.

22. Trilk JL, Singhal A, Bigelman KA, et al. (2011) Effect of sprint interval training on circulatory function during exer- cise in sedentary, overweight/obese women. Eur J Appl Physiol 111: 1591-1597.

23. Ali-Mohamadi M, Abbaspoor M, Rahimi R, et al. (2014) The influence of order execution components of the strength and endurance in the concurrent training on lipid profile and body composition in overweight females. World Appl Sci J 29: 946953.

24. Fisher G (2016) A Role for High Intensity Interval Training (Hiit) for Improving Cardiometabolic Health Outcomes. Obes Open Access 2.2.

25. Forbes GB (2012) Human body composition: Growth, aging, nutrition, and activity. Springer, New York, 343.

26. Kim J, Wang Z, Heymsfield SB, et al. (2002) Total-body skeletal muscle mass: Estimation by a new dual-energy X-ray absorptiometry method. Am J Clin Nutr 76: 378-383.

27. Amarante do Nascimento M, Januário RS, Gerage AM, et al. (2013) Familiarization and reliability of one repetition maximum strength testing in older women. J Strength Cond Res 27: 1636-1642.

28. Ainsworth B, Haskell W, Herrmann S, et al. (2011) 2011 Compendium of Physical Activities: A second update of codes and MET values. Med Sci Sports Exerc 43: 1575-1581.

29. Hedges L (1982) Statistical Methodology in Meta-Analysis. National Institute of Education, Washington DC. Princeton, NJ: ERIC T/M, Educational Testing Service, 17.

30. Thomas JR, Salazar W, Landers DM (1991) What is missing in p less than 0.05? Effect size. Res Q Exerc Sport 62: 344-348.

31. Fock KM, Khoo J (2013) Diet and exercise in management of obesity and overweight. J Gastroenterol Hepatol 28: 59-63.

32. Westcott W, Colligan A, Puhala K, et al. (2017) Exercise and nutrition effects on body composition and blood measures in overweight adults. Journal of Exercise Physiology Online 20: 200-220.

33. Sillanpää E, Häkkinen A, Häkkinen K (2013) Body composition changes by DXA, BIA and skinfolds during exercise training in women. Eur J Appl Physiol 113: 2331-2341.

34. Skelly L, Andrews P, Gillen J, et al. (2014) High-intensity interval exercise induces 24 -h energy expenditure similar to traditional endurance exercise despite reduced time commitment. Appl Physiol Nutr Metab 39: 845-848.

35. Moore DR, Camera DM, Areta JL, et al. (2014) Beyond muscle hypertrophy: Why dietary protein is important for endurance athletes. Appl Physiol Nutr Metab 39: 987-997.

36. Larry Kenney W, Wilmore J, Costill D (2011) Physiology of Sport and Excersise. ( $5^{\text {th }}$ edn), Courier Companies Inc, United States. 